\title{
Chemotherapy followed by chemoradiotherapy in locally advanced pancreatic cancer: A literature review and report of two cases
}

\author{
MARIACRISTINA DI MARCO ${ }^{1,2}$, MARINA MACCHINI ${ }^{1}$, SILVIA VECCHIARELLI $^{1}$, \\ RICCARDO CASADEI $^{3}$, RAFFAELE PEZZILLI ${ }^{4}$, STEFANO FANTI ${ }^{5}$, LUCIA ZANONI ${ }^{5}$, \\ LUCIA CALCULLI $^{6}$, ENZA BARBIERI ${ }^{7}$, DONATELLA SANTINI ${ }^{8}$, \\ ROBERTO DI CICILIA ${ }^{1}$, GIOVANNI BRANDI ${ }^{1}$ and GUIDO BIASCO ${ }^{1,2}$
}

\author{
${ }^{1} \mathrm{~L}$ and A Seràgnoli' Department of Hematology and Oncological Sciences; ${ }^{2}$ Interdepartmental Centre of \\ Cancer Research 'G. Prodi'; Departments of ${ }^{3}$ Surgery, ${ }^{4}$ Digestive Diseases and Internal Medicine, ${ }^{5}$ Nuclear Medicine, \\ ${ }^{6}$ Radiology, ${ }^{7}$ Radiotherapy, and ${ }^{8}$ Pathology, S. Orsola-Malpighi Hospital, University of Bologna, 40138 Bologna, Italy
}

Received September 27, 2010; Accepted December 16, 2010

DOI: $10.3892 / \mathrm{ol} .2011 .253$

\begin{abstract}
The optimal treatment of patients with locally advanced pancreatic cancer remains to be elucidated. Chemoradiotherapy is regarded as the treatment of choice, and studies have examined the sequential schedule of induction chemoradiotherapy followed by chemoradiotherapy, with favourable results. This study investigated the principal clinical trials of chemoradiotherapy treatment in locally advanced pancreatic cancer in 2 patients. The 2 patients received induction chemotherapy with gemcitabine $1000 \mathrm{mg} / \mathrm{mq}$ day on days 1 and 8 of a 21-day cycle for two cycles, followed by chemoradiotherapy with concurrent radiosensitizer bi-weekly gemcitabine $50 \mathrm{mg} / \mathrm{mq}$ for six weeks. Radiotherapy consisted of an external conformational 3D treatment administered to the pancreatic bed and locoregional nodes, with a total dose of 4500 Gy fractionated in $180 \mathrm{~Gy} / \mathrm{day}$, and a boost of $900 \mathrm{~Gy}$ to the neoplastic mass. Efficacy was evaluated four weeks after the end of treatment by a computed tomography (CT) scan and by fluorodeoxyglucose positron-emission tomography/CT. The patients underwent further treatment with periodical instrumental evaluation. A disease control rate was observed in the two patients following sequential treatment, enhanced by subsequent treatment. The two patients remained alive 23-24 months following the diagnosis. The sequential treatment schedule therefore was an effective option in our locally advanced pancreatic cancer patients. A phase III trial and further investigation are required to verify this option in clinical practice.
\end{abstract}

Correspondence to: Dr Mariacristina Di Marco, 'L and A Seràgnoli' Department of Hematology and Oncological Sciences, University of Bologna, Via Massarenti 9, 40138 Bologna, Italy

E-mail: mariacristina.dimarco@unibo.it

Key words: locally advanced pancreatic cancer, chemotherapy, chemoradiotherapy, multimodal treatment

\section{Introduction}

Exocrine pancreatic adenocarcinoma is the fourth most common cause of cancer-related mortality in both genders in the US (1). Surgery is the only potentially curative option but is confined to patients with localized disease. Therapeutic options in patients not eligible for resection are limited to chemotherapy (CHT) or chemoradiotherapy (CRT). However, the prognosis is poor, ranging from 6 to 10 months in locally advanced stage disease.

Locally advanced pancreatic cancer (LAPC) is defined as a tumour localized in the pancreas, without metastatic spread, but directly encasing the adjacent arteries, such as the coeliac axis and superior mesenteric artery, or occluding the superior mesenteric vein, portal vein or mesentericportal vein confluence (2). The optimal treatment of LAPC remains to be elucidated. However, enhancement of clinical benefit, disease control rate (DCR) and survival, and an increase of the resectability rate in borderline resectable disease are crucial. Traditionally, CRT was considered to be the treatment of choice for LAPC. However, despite the known superiority of CRT compared to radiotherapy (RT) alone and best supportive care $(3,4)$, no evidence is available to prove that the beneficial effect of CRT is superior to that of CHT alone (3-9). Although pancreatic tissue exhibits poor radiosensitivity, the addition of $\mathrm{RT}$ is designed to obtain local disease control and an early palliation of symptoms. Numerous studies have tested the sequential schedule of CRT as induction CHT followed by CRT, with favourable results (10-16). Thus, the association of various therapeutic techniques including CRT, surgery and locoregional treatment may be introduced in clinical practice to enhance the DCR in selected patients in multimodal treatment.

In this study, 2 patients with LAPC received a multimodal treatment starting with gemcitabine (GEM)-CHT followed by CRT, with concurrent radiosensitizer bi-weekly GEM, in order to prepare the patients for surgery. 


\section{Patients and methods}

Case 1. In August 2008, a 63-year-old male was diagnosed with locally advanced stage and poorly differentiated carcinoma originating from the head of the pancreas. The Eastern Cooperative Oncology Group (ECOG) performance status was 2. The tumour markers [carcinoembryonic antigen (CEA) and CA19.9] were negative. Since the patient was considered unfit for surgery, the tumour was treated medically. A sequential CHT followed by CRT was scheduled in September 2008. However, the treatment was discontinued due to toxicity, in the form of fever and nausea, during the induction CHT period. In February 2009, an abdominal computed tomography (CT) scan showed a significant reduction of the pancreatic tumour mass and a fluorodeoxyglucose positron-emission tomography/CT $\left({ }^{18} \mathrm{CT}\right.$-PET) revealed a complete metabolic response (Fig. 1). Consequently, the patient underwent surgery. Since the tumour involved the coeliac axis and superior mesenteric artery, surgery was confined to explorative laparotomy. In July 2009, a CT scan showed a further reduction of tumour size, and ${ }^{18} \mathrm{CT}$-PET confirmed the complete metabolic response. The ECOG performance status was 0 . In agreement with the patient, treatment involved the maintenance of GEM at $1000 \mathrm{mg} / \mathrm{mq}$ on days 1,8 and 15 of a 28-day cycle from July 2009 to February 2010, when a single hepatic metastasis developed, 18 months following the LAPC diagnosis. A slight increase in CEA was found $(6.7 \mathrm{ng} / \mathrm{ml})$. Thus, CHT with gemcitabine and oxaliplatin (GEMOX schedule) commenced from March 2010. During treatment, a case of grade 3 neutropenia occurred.

Case 2. In July 2008, a 63-year-old male was diagnosed with a locally advanced adenocarcinoma of the pancreatic body. The ECOG performance status was 2. The tumour marker CA19.9 was $143 \mathrm{U} / \mathrm{ml}$. The patient was considered unfit for surgery. Consequently, the tumour was medically treated. From July to October 2008 a sequential CHT followed by CRT was performed without major toxicities. In November 2008, a CT scan revealed a slight reduction in tumour size $(30$ vs. $39 \mathrm{~mm}$ ), but coeliac axis tumour involvement persisted. Since the CA19.9 value remained high, the patient was treated with CHT according to the GEMOX schedule from January to September 2009. Further tumour shrinkage ( 28 vs. $39 \mathrm{~mm}$ ) and a complete metabolic response were noted. The CA19.9 value decreased to $29 \mathrm{U} / \mathrm{ml}$. In November 2009, the patient underwent radiofrequency ablation (RFA) with a positive locoregional response and a normalization of CA19.9 serum values. In March 2010, the patient resumed CHT according to the GEMOX schedule due to an increase in CA19.9 (345 $\mathrm{U} / \mathrm{ml}$ ), despite negative a ${ }^{18} \mathrm{CT}$-PET evaluation. CHT was discontinued in May 2010 and a CT scan revealed stable disease: serum CA19.9 levels had decreased to $135 \mathrm{U} / \mathrm{ml}$ and ${ }^{18} \mathrm{CT}$-PET showed minimal pathological uptake of the tracer at the mesenteric node and in the lung nodules.

\section{Results and Discussion}

This study evaluated clinical cases of patients characterized by long-term DCR and metabolic tumour deactivation, as noted by a negative ${ }^{18} \mathrm{CT}$-PET, obtained first by the early sequential CRT schedule, and followed by further treatment modality
(Figs. 1 and 2). Patient 1 developed a single hepatic metastasis 18 months after LAPC diagnosis, following upfront sequential CRT and the first line CHT. The patient remained alive at 23 months and in good clinical condition. RFA appeared to prolong disease control in patient 2 . This patient developed metastasis 24 months after the LAPC diagnosis. The two patients therefore experienced clinical benefits.

It is known that the optimal treatment of patients with LAPC has yet to be elucidated. CHT alone is the treatment of choice in the UK, whereas CRT is the standard modality in the USA. Numerous trials have confirmed the enhanced efficacy of the CRT, although the survival benefit of combination treatment appears to be moderate (Table I). Discrepancies in the results of two phase III studies were noted $(8,9)$. Loehrer et al showed a slightly longer survival in patients treated with CRT with concurrent GEM vs. GEM alone (11 vs. 9.4 months, $\mathrm{p}=0.044)$ (8). On the other hand, the Fédération Francophone de Cancérologie digestive (FFCD) and the Société Francophone de Radiothérapie Oncologique (SFRO) study proved that GEM was more favourable than CRT with 5-fluorouracil and cisplatin followed by GEM (13 vs. 8.6 months, $\mathrm{p}=0.03$ ) (9).

In the last decade, CHT and RT have been tested in a sequential modality comprising an upfront mono- or multichemotherapy, followed by CRT, or maintenance CHT (Table II). The most significant data on the efficacy of induction CHT, derived from two large retrospective analyses and a phase II trial, proved the superiority of the sequential regimen compared to CRT or CHT. Krishnan et al (11) showed increased overall survival (OS) in patients pretreated with GEM-based CHT and CRT compared to patients who received only CRT (OS: 11.9 vs. 8.5 months, p<0.001; PFS: 6.4 vs. 4.2 months, $\mathrm{p}<0.001)$. On the other hand, the GERCOR analysis showed an OS of 15 months in patients pretreated with various CHT regimens prior to CRT with concurrent fluoropyrimidine, whereas OS was only 11 months in the CHT-only arm $(\mathrm{p}=0.0009)$ (12). In a phase II trial, Hudson et al (15), confirmed the positive effect of upfront CHT prior to CRT, achieving an OS of 15.3 months compared to 9.2 months in frontline CRT $(\mathrm{p}=0.005)$.

No consensus exists on an ideal schedule for sequential treatment. We used GEM-based CHT in the systemic induction phase as compared to the CRT phase $(10,11,13-16)$, whereas in the majority of studies the concurrent drug in the CRT phase was 5-fluorouracil or derivates thereof (11-13,16). Although the survival improvement was poor, the tumour resectability rate was increased by RT with concurrent GEM vs 5-fluorouracil in LAPC (17). No certain dose of GEM with concurrent RT $(8,10,11,14-16)$ was defined; during conventional radiation the safe weekly dose of GEM should remain at $<400 \mathrm{mg} / \mathrm{mq}$, whereas this dose is not effective for systemic disease control. Instead, certain studies tested a lower dosage of GEM twice weekly as a radiosensitizer enhancer with positive results. For the same purpose, we treated our patients with bi-weekly $50 \mathrm{mg} / \mathrm{mq}$ concurrent to RT (18).

The rationale of sequential CRT is based on the early metastatic spread of LAPC: patients with disease that is still localized are likely to complete treatment with CRT, whereas patients with disease progression during the induction CHT may benefit more from a different $\mathrm{CHT}$ regimen. Theoretically the first CHT phase is utilized to select patients on the basis of 


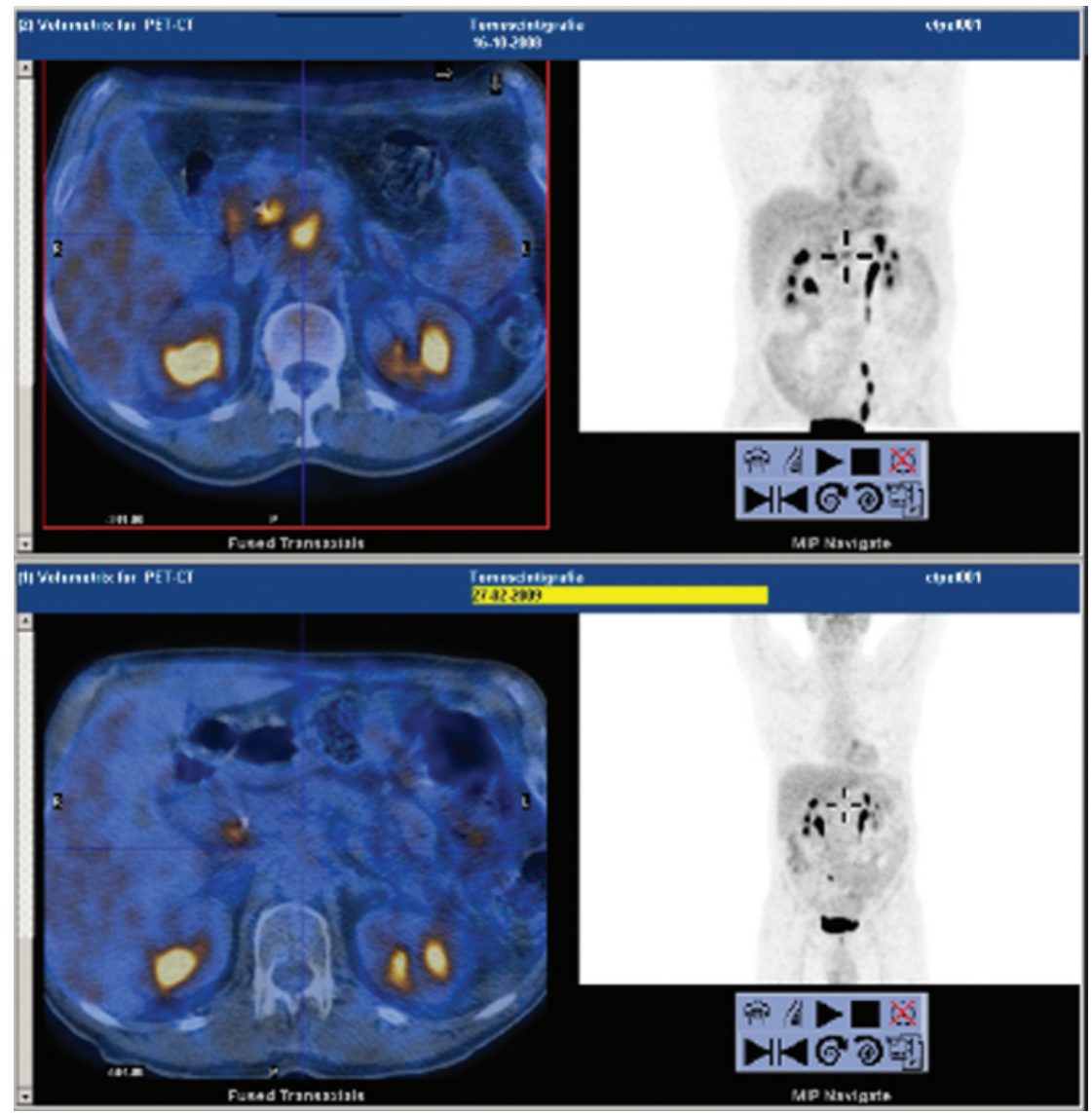

Figure $1 .{ }^{18} \mathrm{CT}$-PET evaluation prior to and following chemoradiotherapy treatment in patient 1.

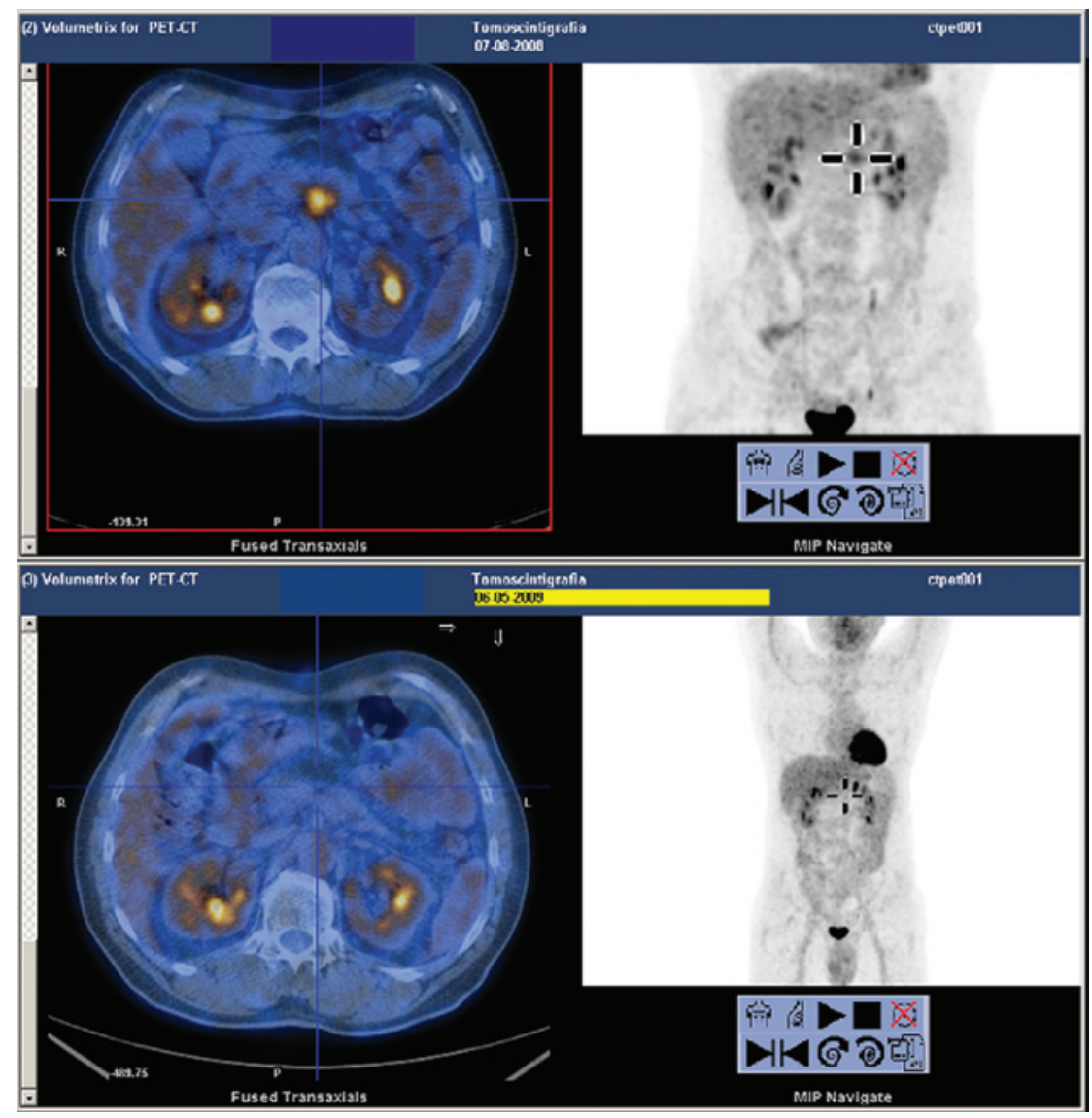

Figure $2 .{ }^{18} \mathrm{CT}$-PET evaluation prior to and following chemoradiotherapy treatment in patient 2. 


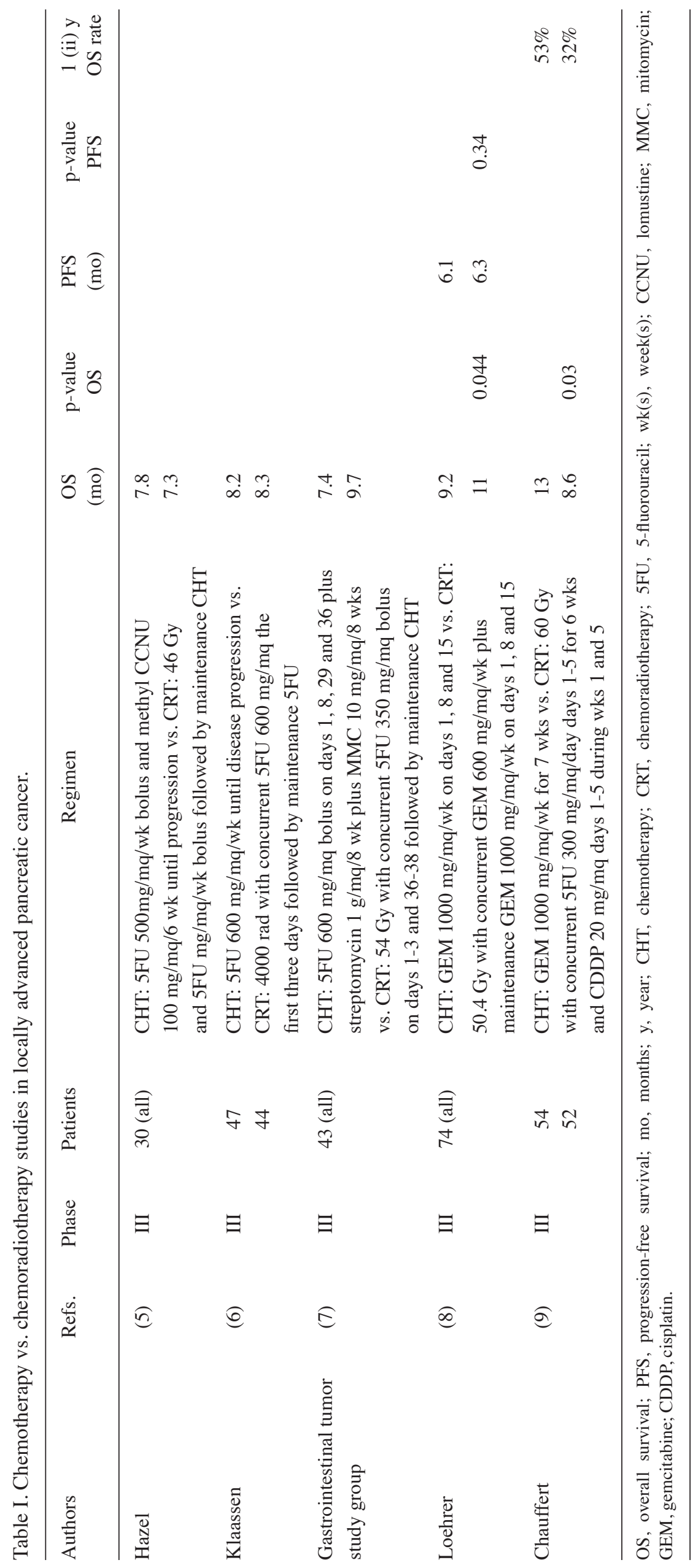




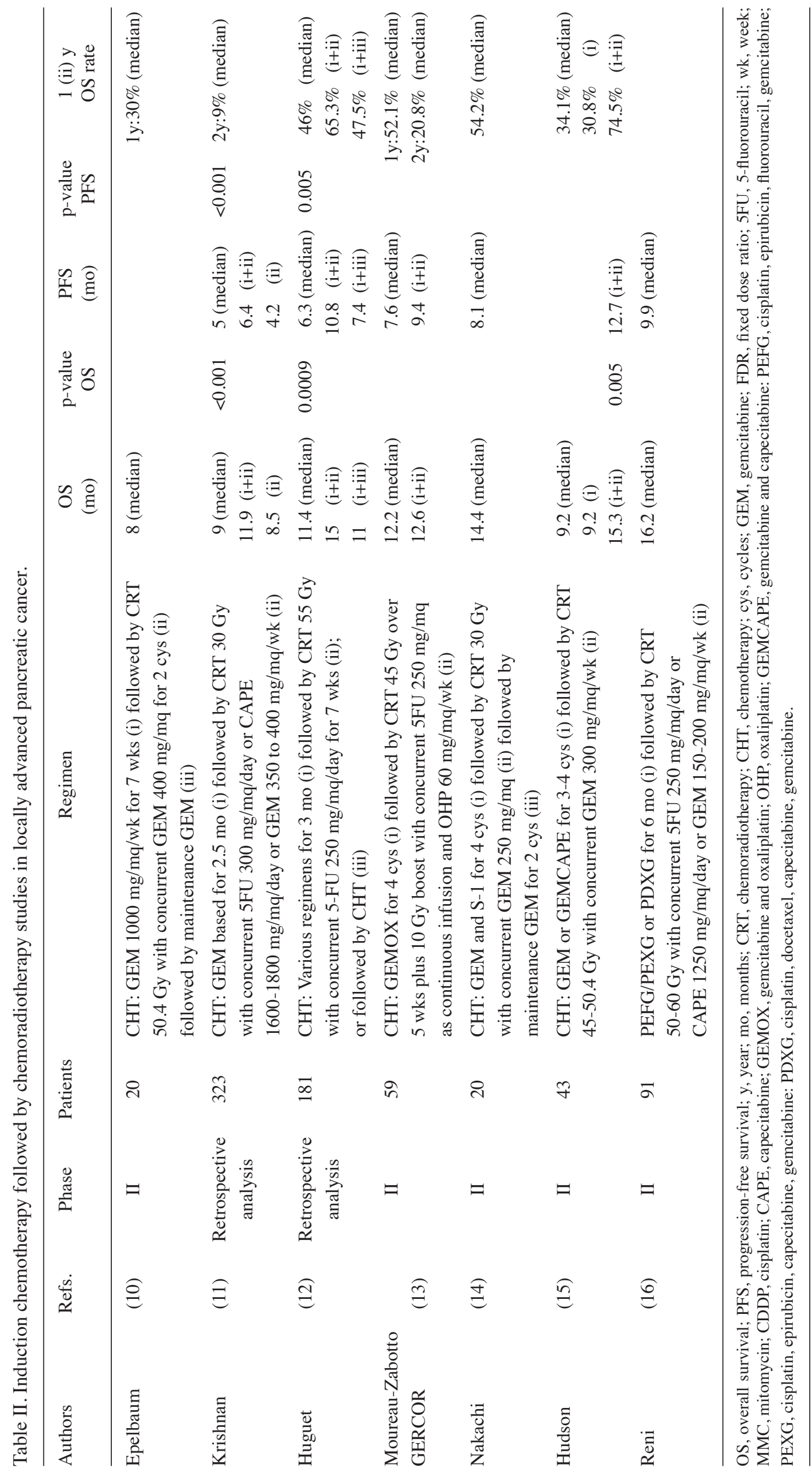


their biological disease malignancy, and adopt the therapeutic strategy best tailored to the individual patient. The same rationale can be extended to the neo-adjuvant setting in that despite the failure of neo-adjuvant intent in our patients, certain authors promote the sequential schedule since it appears to enhance the selection of patients with borderline resectable LAPC $(10,16,17)$. This promotion is crucial in increasing secondary resectability rate, when the bulk of the disease can be reduced, and avoiding surgery in patients becoming rapidly metastatic.

Conversely, induction CHT followed by CRT in our patients appeared to enhance the clinical benefit rate and DCR when combined with further treatment. In particular, the addition of RFA appeared to extend the metastasis-free survival time to 24 months in patient 2 , although these data have yet to be elucidated in the available literature $(19,20)$. To the best of our knowledge, the multimodal treatment may be considered in selected patients who obtain a protracted stable disease due to a number of treatment techniques employed at various stages of disease.

Our cases on sequential CHT and CRT may serve as a useful starting point for critical consideration. However, numerous issues have yet to be elucidated. These issues include which chemotherapeutic scheme is ideal in the induction phase and the amount of drugs to be administered; the length of time for which patients should receive frontline CHT to improve the selection for CRT; which drugs should be administered concurrently with radiotherapy; as well as the best dosage and purpose. Therefore, phase III studies are required to better define the role of sequential CHT and CRT in LAPC patients since patients with borderline resectable cancer may benefit from better selection for surgery, whereas patients with unresectable disease may experience enhanced DCR.

\section{References}

1. Jemal A, Siegel R, Ward E, Hao Y, Xu J and Thun MJ: Cancer Statistics. CA Cancer J Clin 59: 225-249, 2009.

2. Varadhachary GR, Tamm EP, Abbruzzese JL, Xiong HQ, Crane CH, Wang H, Lee JE, Pisters PW, Evans DB and Wolff RA: Borderline resectable pancreatic cancer: definitions, management, and role of preoperative therapy. Ann Surg Oncol 13: 1035-1046, 2006.

3. Yip D, Karapetis C, Strickland A, Steer CB and Goldstein D: Chemotherapy and radiotherapy for inoperable pancreatic cancer. Cochrane Database Syst Rev 3: CD002093, 2006.

4. Sultana A, Tudur Smith C, Cunningham D, Starling N, Tait D, Neoptolemos JP and Ghaneh P: Systematic review, including meta-analysis, on the management of locally advanced pancreatic cancer using radiation/combined modality therapy. Brit $\mathrm{J}$ Cancer 96: 1183-1190, 2007.

5. Hazel JJ, Thirlwell MP, Huggins M, Maksymiuk A and MacFarlane JK: Multi-drug chemotherapy with and without radiation for carcinoma of the stomach and pancreas: a prospective randomized trial. J Can Assoc Radiol 32: 164-165, 1981.

6. Klaassen DJ, MacIntyre JM, Catton GE, Engstrom PF and Moertel CG: Treatment of locally unresectable cancer of the stomach and pancreas: a randomized comparison of 5-fluorouracil alone with radiation plus concurrent and maintenance 5-fluorouracil - an Eastern Cooperative Oncology Group study. J Clin Oncol 3: 373-378, 1985.
7. Gastrointestinal tumour study group: Treatment of locally unresectable carcinoma of the pancreas: comparison of combined-modality therapy (chemotherapy plus radiotherapy) to chemotherapy alone. J Natl Cancer Inst 80: 751-755, 1988.

8. Loehrer PJ, Powell ME, Cardenes HR, Wagner L, Brell JM, Ramanathan RK, Crane CH, Alberts SR, Benson AB and Eastern Cooperative Oncology Group: A randomized phase III study of gemcitabine in combination with radiation therapy versus gemcitabine alone in patients with localized, unresectable pancreatic cancer: E4201. J Clin Oncol 26: 214s (suppl), 2008.

9. Chauffert B, Mornex F, Bonnetain F, et al: Phase III trial comparing intensive induction chemoradiotherapy (60 Gy, infusional 5-FU and intermittent cisplatin) followed by maintenance gemcitabine with gemcitabine alone for locally advanced unresectable pancreatic cancer. Definitive results of the 2000-01 FFCD/SFRO study. Ann Oncol 19: 1592-1599, 2008.

10. Epelbaum R, Rosenblatt E, Nasrallah S, Faraggi D, Gaitini D, Mizrahi S and Kuten A: Phase II study of gemcitabine combined with radiation therapy in patients with localized, unresectable pancreatic cancer. J Surg Oncol 81: 138-143, 2002.

11. Krishnan S, Rana V, Janjan NA, Varadhachary GR, Abbruzzese JL, Das P, Delclos ME, Gould MS, Evans DB, Wolff RA and Crane $\mathrm{CH}$ : Induction chemotherapy selects patients with locally advanced, unresectable pancreatic cancer for optimal benefit from consolidative chemoradiation therapy. Cancer 110: 47-55, 2007.

12. Huguet F, André T, Hammel P, Artru P, Balosso J, Selle F, Deniaud-Alexandre E, Ruszniewski P, Touboul E, Labianca R, de Gramont A and Louvet C: Impact of chemoradiotherapy after disease control with chemotherapy in locally advanced pancreatic adenocarcinoma in GERCOR phase II and III studies. J Clin Oncol 25: 326-331, 2007.

13. Moureau-Zabotto L, Phélip JM, Afchain P, et al: Concomitant administration of weekly oxaliplatin, fluorouracil continuous infusion, and radiotherapy after 2 months of gemcitabine and oxaliplatin induction in patients with locally advanced pancreatic cancer: a Group Coordinateur Multidisciplinaire en Oncologie Phase II Study. J Clin Oncol 26: 1080-1085, 2008.

14. Nakachi K, Furuse J, Kinoshita T, Kawashima M, Ishii H, Ikeda M, Mitsunaga S and Shimizu S: A phase II study of induction chemotherapy with gemcitabine plus S-1 followed by chemoradiotherapy for locally advanced pancreatic cancer. Cancer Chemother Pharmacol 66: 527-534, 2010.

15. Hudson E, Hurt C, Mort D, Brewster AE, Iqbal N, Joseph G, Crosby TD and Mukherjee S: Induction chemotherapy followed by chemoradiation in locally advanced pancreatic cancer: an effective and well-tolerated treatment. Clin Oncol 22: 27-35, 2010.

16. Reni M, Cereda S, Balzano G, Passoni P, Rognone A, Zerbi A Nicoletti R, Mazza E, Arcidiacono PG, Di Carlo V and Villa E: Outcome of upfront combination chemotherapy followed by chemoradiation for locally advanced pancreatic adenocarcinoma. Cancer Chemother Pharmacol 64: 1253-1259, 2009.

17. Crane $\mathrm{CH}$, Abbruzzese JL, Evans DB, et al: Is the therapeutic index better with gemcitabine-based chemoradiation than with 5-fluorouracil-based chemoradiation in locally advanced pancreatic cancer? Int J Radiat Oncol Biol Phys 52: 1293-1302, 2002.

18. Magnino A, Gatti M, Massucco P, Sperti E, Faggiuolo R, Regge D, Capussotti L, Gabriele P and Aglietta M: Phase II trial of primary radiation therapy and concurrent chemotherapy for patients with locally advanced pancreatic cancer. Oncology 68: 493-499, 2005.

19. Girelli R, Frigerio I, Salvia R, Barbi E, Tinazzi Martini P and Bassi C: Feasibility and safety of radiofrequency ablation for locally advanced pancreatic cancer. Br J Surg 97: 220-225, 2010.

20. Casadei R, Ricci C, Pezzilli R, Serra C, Calculli L, MorselliLabate AM, Santini D and Minni F: A prospective study on radiofrequency ablation locally advanced pancreatic cancer. Hepatobiliary Pancreat Dis Int 9: 306-311, 2010. 